\title{
EXTRAÇÃO DE INFORMAÇÕES DE LAUDOS MÉDICOS UTILIZANDO TEXT MINING: APLICACÇÃO DO MÉTODO WEBIBLIOMINING
}

\author{
Isaac de Abreu GASPAR ${ }^{1,2 *}$, Aldo SHIMOYA ${ }^{2} \&$ Eduardo SHIMODA ${ }^{2}$
}

1 Instituto federal Fluminense. Campos dos Goytacazes, Rio de Janeiro, Brasil.

2 Universidade Candido Mendes. Campos dos Goytacazes, Rio de Janeiro, Brasil.

*Autor para correspondência: iagaspar25@gmail.com

DOI: http://dx.doi.org/10.18571/acbm.169

\section{RESUMO}

Este trabalho apresenta um modelo de mineração de fonte de dados bibliográficas conhecido como webibliomining, abordando a aplicação de técnicas de mineração de textos (text mining) em laudos médicos. O objetivo é a aplicação do modelo na realização de um levantamento bibliométrico, realizado na base Scopus. A chave de busca utilizada foi “"Text Mining' AND 'Medical Reports Information'", retornando 82 registros de publicações. Os artigos mais relevantes foram considerados para compor o núcleo de partida para a pesquisa bibliográfica, pesquisa esta que demonstra e comprova as aplicações efetivas e satisfatórias da utilização da mineração de texto aplicadas a laudos médicos.

Palavras-chave: Text Mining; Laudos médicos; Webibliominig.

\begin{abstract}
This work presents a mining model of bibliographic data source known as webibliomining, addressing the application of text mining techniques in medical reports. The objective is the application of the model in the accomplishment of a bibliometric survey, carried out in the base Scopus. The search key used was "'Text Mining' AND 'Medical Reports Information'", returning 82 publication records. The most relevant articles were considered to compose the starting nucleus for the bibliographic research, a research that demonstrates and proves the effective and satisfactory applications of the use of text mining applied to medical reports.
\end{abstract}

Keywords: Text Mining; Medical Reports; Webibliominig.

\section{Introdução}

A mineração de textos, ou text mining, é uma técnica utilizada para a manipulação automática de grandes volumes de dados, pertencente ao campo da ciência da computação, e está cientificamente ligada ao desenvolvimento de ferramentas de recuperação automática da informação. A mineração de textos explora textos e documentos buscando identificar termos relevantes assim como estabelecer padrões textuais e desenvolver grupos temáticos de assuntos pela frequência de aparecimento de termos no domínio a ser analisado.

Segundo Costa (2010), o processo de descoberta de conhecimento textual é composto pelas seguintes etapas:

I. Etapa de Pré-processamento - Esta etapa tem a finalidade de preparar, transformar, organizar e melhorar a qualidade do texto para a etapa seguinte;

II. Etapa de Processamento - É nesta etapa que ocorre a mineração propriamente dita. As técnicas de mineração de texto são aplicadas de acordo com a finalidade pretendida, 
entre elas a recuperação da informação, indexação, extração da informação, associação de documentos, sumarização, clusterização e classificação/categorização;

III. Etapa de Pós-processamento - Esta etapa analisa os resultados obtidos na etapa de processamento.

As etapas descritas foram derivadas do modelo KDD (Knowledge Discovery in Databases) utilizado na mineração de dados, cujo processo é baseado em dados estruturados. Diferentemente, a mineração de textos busca extrair padrões relevantes de textos não estruturados (COSTA, 2010). Diversos trabalhos dissertam acerca da aplicação das técnicas de mineração de textos em laudos médicos, entretanto poucas pesquisas buscaram realizar uma revisão bibliográfica sistemática da produção científica nesta área do conhecimento.

Segundo Conforto (2011), a revisão bibliográfica desenha uma linha limítrofe na pesquisa que se deseja desenvolver, sob uma perspectiva científica, e que para isso é preciso definir os tópicos chave, autores, palavras, periódicos e fontes de dados preliminares.

Diante do exposto, optou-se por realizar uma revisão sistemática da produção científica acerca da utilização de técnicas de mineração de textos para extrair informações de laudos médicos. O objetivo deste trabalho é criar uma referência para que pesquisadores possam ter um ponto de partida em uma pesquisa sobre o tema discutido.

\subsection{Webibliomining}

Segundo Costa (2010), a webibliomining integra conceitos de bibliometria (bibliometrics), webmetria (webmetrics), informetria (informetrics) e mineração bibliográfica (biblioming), tendo por objetivo apoiar definição de um conjunto inicial de referências bibliográficas para o desenvolvimento de pesquisas.

A webibliomining proporciona uma investigação entre as referências, o que resulta em um estudo preliminar contendo trabalhos que farão parte do "núcleo de partida", garantindo ao pesquisador embasamento suficiente para o próprio estudo bibliográfico.

O método considera a execução das seguintes etapas (COSTA, 2010):

I. Definição da amostra da pesquisa;

II. Pesquisa na amostra, com as palavras-chave;

III. Identificação dos periódicos com maior número de artigos publicados sobre o tema;

IV. Identificação dos autores com maior número de publicações;

V. Levantamento da cronologia da produção, identificando os ciclos de maior produção;

VI. Seleção dos artigos para a composição do "núcleo de partida" para a pesquisa bibliográfica.

\section{Materiais e Métodos}

O modelo foi aplicado neste trabalho a fim de definir um conjunto inicial de referências bibliográficas para uma investigação mais profunda sobre o tema "Mineração de textos aplicada a laudos médicos".

A amostra pesquisada corresponde aos artigos indexados na base Scopus. A escolha desta base de dados se deu pela grande abrangência das mesmas em todas as áreas do conhecimento, anexando assim, tanto periódicos quanto patentes, livros e artigos de congressos. Neste trabalho foi considerado somente as publicações do tipo "Artigo". A pesquisa foi realizada em junho de 2017. A fim de avaliar as publicações mais recentes, o período de pesquisa foi reduzido para retornar apenas os artigos publicados no período de 2012 a 2017. 
A chave de busca utilizada foi “ 'Text Mining' AND 'Medical Reports Information' ”. No campo "pesquisa avançada", selecionou-se o filtro que mostrou apenas os resultados que continham estes termos no resumo, palavras-chave ou título.

Levando-se em conta os resultados obtidos na busca, foi elencado somente os periódicos que possuíam pelo menos três artigos publicados em sua base.

Em seguida, verificou-se a distribuição de registros quanto à autoria dos artigos. Devido ao grande número de autores, elencou-se apenas os 10 autores que mais publicaram na plataforma. Nesta pesquisa tratou-se da mesma forma a autoria e a coautoria.

Em sequência, foi abordada a soma de todos os textos publicados no decorrer dos anos sobre o assunto tratado, apresentando-se os dados referentes à distribuição de registros quanto ao ano de publicação, de forma que se possa observar a evolução da produção científica no tema pesquisado, em uma escala cronológica. Para isso, considerou-se todos os anos em que houve publicação na plataforma sobre o tema estudado.

Realizou-se também um levantamento da produção por país, a fim de verificar o grau de importância dada ao tema em diferentes partes do mundo. Considerou-se apenas países com 2 ou mais publicações.

Para a escolha de uma bibliografia básica sobre este assunto, foi selecionado um conjunto de artigos que será tratado como núcleo de partida para uma revisão de literatura dentro dos termos pesquisados. Para isso, abriu-se o resumo de cada artigo encontrado, considerando-se como artigos mais relevantes apenas os que tratavam do tema da utilização de técnicas de mineração de textos aplicadas à laudos médicos.

\section{Resultados}

\subsection{Definição da Amostra}

A consulta na base Scopus retornou 82 registros de artigos publicados no período de 2012 a 2017, buscando-se apenas por documentos do tipo “Artigo”, conforme Quadro 1.

\begin{tabular}{|c|}
\hline "'Free Software' AND 'Public Administration"” \\
\hline Tipo de material pesquisado: Artigos \\
\hline Documentos encontrados: 82 \\
\hline Base de pesquisa: Scopus \\
\hline Cronologia: 2012 a 2017 \\
\hline
\end{tabular}

Quadro 1. Resultado da pesquisa na amostra.

\subsection{Identificação dos Periódicos}

Foram encontrados 60 registros de periódicos indexados na base Scopus com publicações no tema pesquisado. Observa-se na Figura 1 que o periódico Journal of Biomedical Informatics é o que possui o maior número de publicações. Considerou-se apenas os periódicos que possuiam 3 ou mais artigos publicados. 


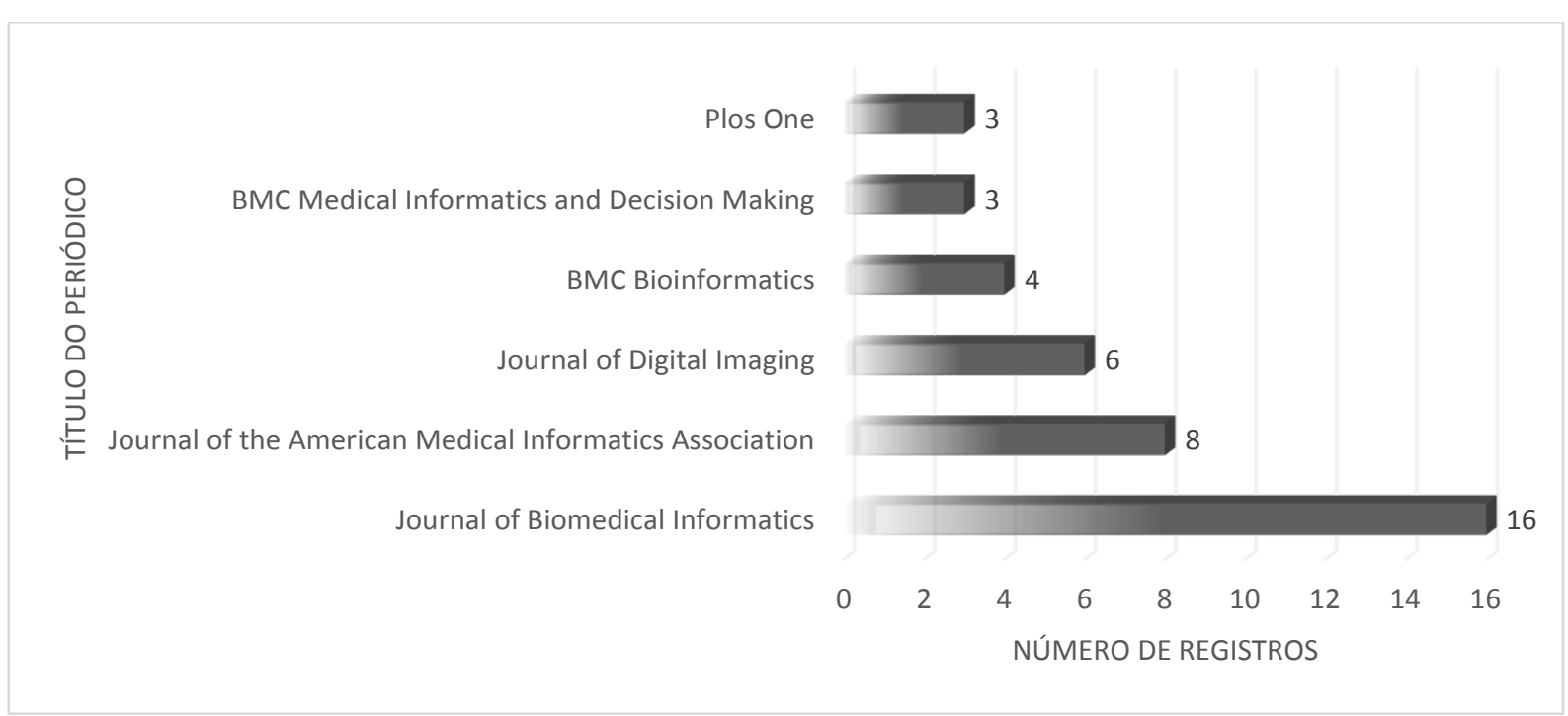

Figura 1: Distribuição de registros por veículo de publicação.

\subsection{Identificação dos Autores}

Na Figura 2 encontra-se a relação dos autores que mais publicaram na base no tema pesquisado. Entre os autores destaca-se Langlotz, C.P., com cinco publicações. Devido ao grande número de autores, elencou-se apenas os 10 autores que mais publicaram na plataforma.

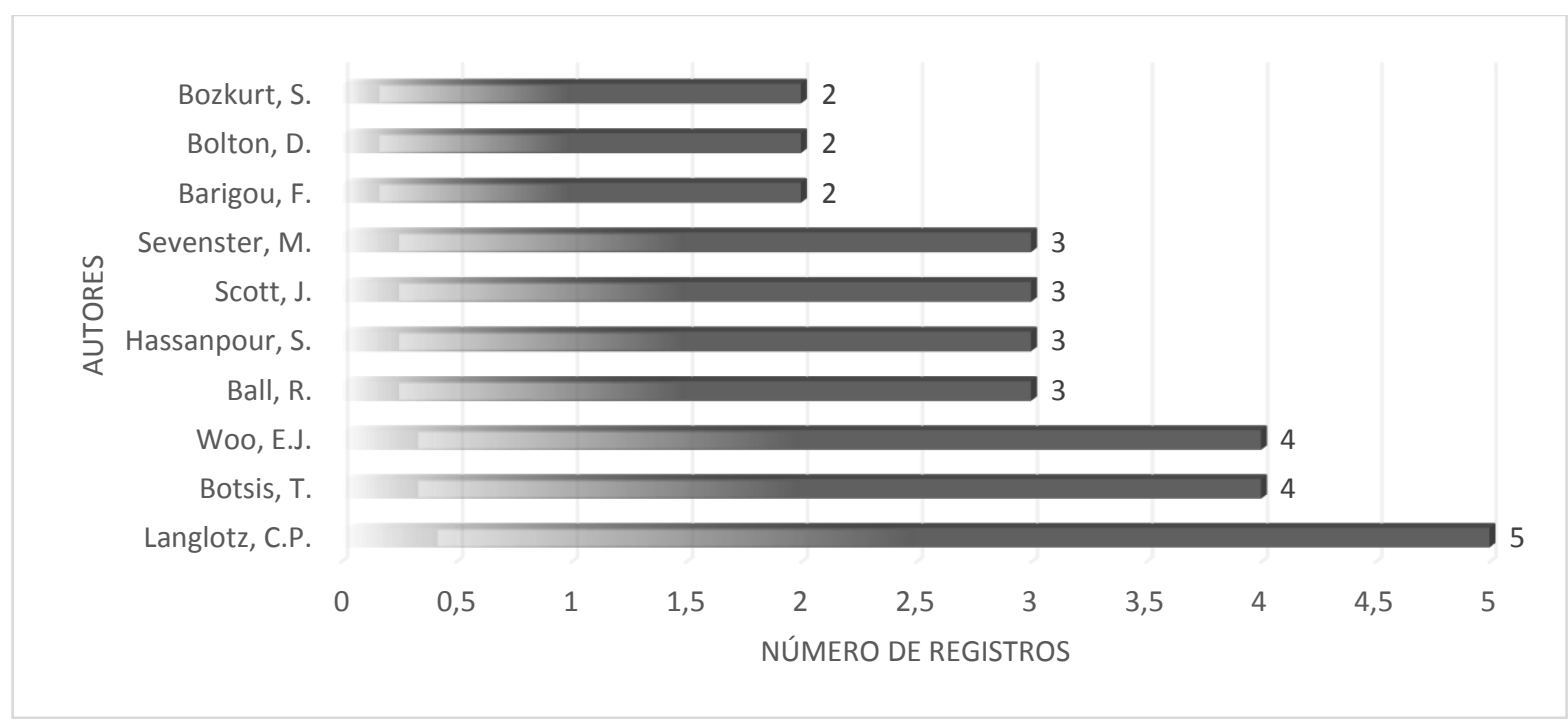

Figura 2: Distribuição de registros de artigo por autor.

\subsection{Levantamento da Cronologia da Produção}

Nesta etapa demonstram-se os resultados da pesquisa em uma escala cronológica. Analisando-se os dados referentes ao ano de publicação, encontrados na Figura 3, observa-se que:

I. O artigo mais antigo indexado na base refere-se ao ano de 1999;

II. Houve um ciclo de produção mais acentuado no período de 2015 e 2016;

III. Não houve produção na base no ano 2000. 


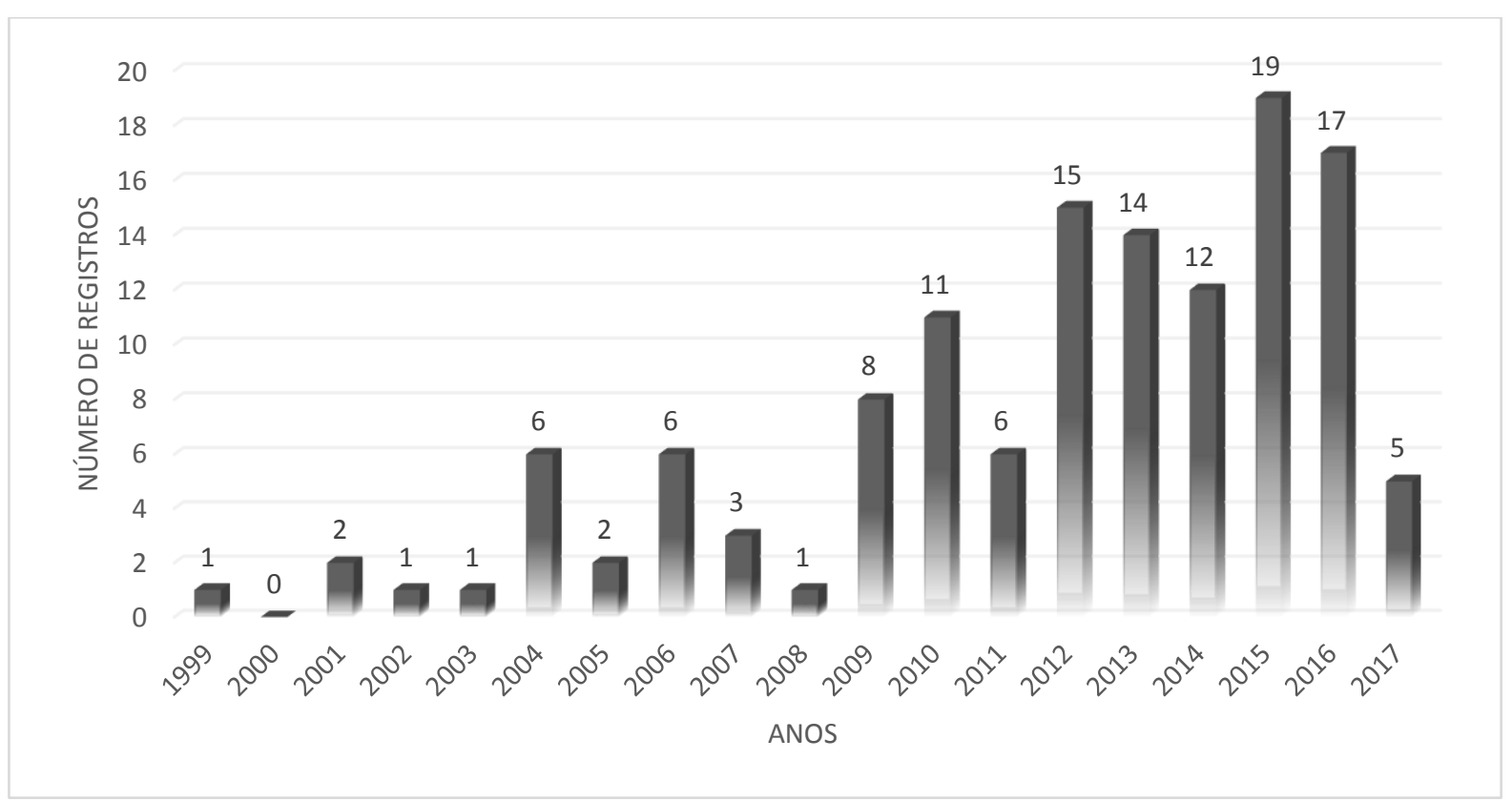

Figura 3: Distribuição de registros por ano de publicação.

\subsection{Levantamento da Produção por País}

Verifica-se na Figura 4 o levantamento da produção por país. Destaca-se na análise os Estados Unidos, que possui o maior número de produções sobre o tema pesquisado. Considerouse apenas países com 2 ou mais publicações.

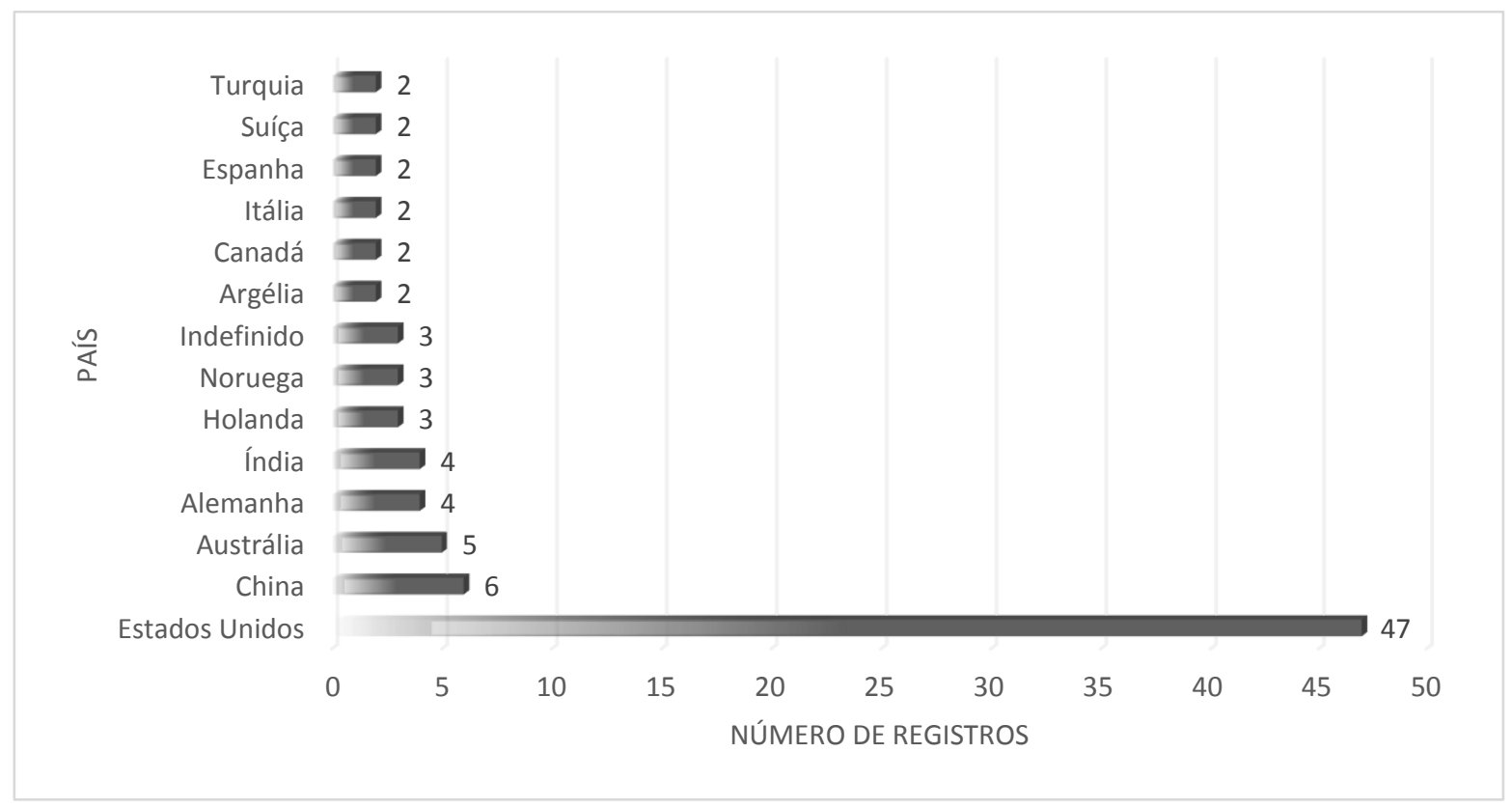

Figura 4: Distribuição de registros por país.

\subsection{Identificação dos Artigos Mais Relevantes}

Pode-se observar no Quadro 2 a lista dos artigos selecionados para compor o "núcleo de partida" para a pesquisa. Neste quadro constam os artigos de maior relevância encontrados na base. Considerou-se como relevantes os artigos que tratavam do tema da utilização de técnicas de mineração de textos para extração de informações de laudos médicos. 


\begin{tabular}{|c|c|}
\hline $\begin{array}{l}\text { ROY, Partha Pratim et al. Keyword spotting in doctor's handwriting on medical } \\
\text { prescriptions. Expert Systems With Applications, v. 76, p.113-128, jun. } 2017 .\end{array}$ & $\begin{array}{l}\text { A DAVIS, Matthew et al. Preliminary analysis of in utero low-level arsenic exposure and fetal } \\
\text { growth using biometric measurements extracted from fetal ultrasound reports. Environmental Health, } \\
\text { v. } 14, \text { n. } 1, \text { p. } 49-53,2015 \text {. }\end{array}$ \\
\hline $\begin{array}{l}\text { HASSANPOUR, Saeed et al. Performance of a Machine Learning Classifier of Knee } \\
\text { MRI Reports in Two Large Academic Radiology Practices: A Tool to Estimate } \\
\text { Diagnostic Yield. American Journal of Roentgenology, v. 208, n. 4, p.750-753, abr. } \\
2017 .\end{array}$ & $\begin{array}{l}\text { MARTíNEZ, David S. Hermina et al. Cross-hospital portability of information extraction of cancer } \\
\text { staging information. Artificial Intelligence in Medicine, Parkville, v. 62, n. 1, p.11-21, } 2014 .\end{array}$ \\
\hline $\begin{array}{l}\text { KOCBEK, Simon et al. Text mining electronic hospital records to automatically classify } \\
\text { admissions against disease: Measuring the impact of linking data sources. Journal of } \\
\text { Biomedical Informatics, v. 64, p.158-167, dez. } 2016 \text {. }\end{array}$ & $\begin{array}{l}\text { Ifonso; MAGR0, Rosario; VITABILE, Salvatore. A text based indexing system for } \\
\text { mage retrieval and classification. Future Generation Computer Systems, Palermo, } \\
\text {,jul. } 2014 .\end{array}$ \\
\hline $\begin{array}{l}\text { BOZKURT, Selen et al. Using automatically extracted information from mammography } \\
\text { reports for decision-support. Journal of Biomedical Informatics, v. 62, p.224-231, ago. } \\
2016 \text {. }\end{array}$ & $\begin{array}{l}\text { LU0, Yuan et al. Automatic lymphoma classification with sentence subgraph mining from pathology } \\
\text { reports. Journal of the American Medical Informatics Association, Cambridge, v. 21, n. 5, p.824- } \\
832,2014 \text {. }\end{array}$ \\
\hline $\begin{array}{l}\text { HASSANPOUR, Saeed; LANGLOTZ, Curtis P.. Information extraction from multi- } \\
\text { institutional radiology reports. Artificial Intelligence in Medicine, v. 66, p.29-39, jan. } \\
2016 .\end{array}$ & $\begin{array}{l}\text { BOTSIS, Taxiarchis; W00, Emilyjane; BALL, RobertF.. The Contribution of the Vaccine Adverse } \\
\text { Event Text Mining System to the Classification of Possible Guillain-Barré Syndrome Reports. } \\
\text { Applied Clinical Informatics, Rockville, v. 4, n. 1, p.88-99, } 2013 .\end{array}$ \\
\hline $\begin{array}{l}\text { CAI, Tianrun et al. Natural Language Processing Technologies in Radiology Research } \\
\text { and Clinical Applications. Radiographics, v. 36, n. 1, p.176-191, jan. } 2016 .\end{array}$ & $\begin{array}{l}\text { SERAPIÃ0, Paulo Roberto Barbosa et al. Construction of an Index of Information From Clinical } \\
\text { Practice in Radiology and Imaging Diagnosis. Radiologia Brasileira, Ribeirão Preto, v. 46, n. } 5 \text {, } \\
\text { p.290-298, set. 2013. }\end{array}$ \\
\hline $\begin{array}{l}\text { RANI, Johanna Johnsi; GLADIS, Dennis; MAMMEN, Joy John. Association analysis of } \\
\text { breast cancer data in pathology reports. International Journal of Applied Engineering } \\
\text { Research, Chennai, v. 10, n. 69, p.123-128, 2015. }\end{array}$ & $\begin{array}{l}\text { IMLER, Timothy D. et al. Natural Language Processing Accurately Categorizes Findings From } \\
\text { Colonoscopy And Pathology Reports. Clinical Gastroenterology and Hepatology, Indianapolis, v. } \\
\text { 11, n. 6, p.689-694, jun. 2013. }\end{array}$ \\
\hline
\end{tabular}

Quadro 2: Artigos mais relevantes encontrados na pesquisa.

\subsection{Resumos dos Artigos Mais Relevantes}

Nesta seção serão apresentados alguns resumos das publicações consideradas de maior relevância para o tema proposto, considerando para isto, os resultados das etapas anteriores.

\subsubsection{Association Analysis of Breast Cancer Data in Pathology Reports}

Este artigo revela que a Índia ocupa o primeiro lugar no ranking mundial de mortes causadas pelo câncer de mama. Este estudo classifica os laudos eletrônicos arquivados em hospitais como recursos valiosos para compreender a gravidade da doença entre os pacientes e para verificar a exatidão do diagnóstico.

Um sistema foi desenvolvido como parte desse trabalho. A base de dados consiste em 150 laudos de um hospital da região. As informações extraídas dos laudos podem ser classificadas como Tumor, Gânglios Linfáticos ou Metástase, que constituem os componentes do protocolo de classificação TNM do American Joint Committee on Cancer (AJCC), que ajuda na determinação do estágio do câncer de pacientes.

O trabalho tem relevância e aplicabilidade prática, considerando o grau de importância da doença no subcontinente.

\subsubsection{Preliminary Analysis of in Utero Low-Level Arsenic Exposure and Fetal Growth Using Biometric Measurements Extracted From Fetal Ultrasound Reports}

O trabalho realizado por Davis, M.A., et. al., utilizou a mineração de textos em 272 relatórios de ultrassonografia fetal encontrados em registros médicos eletrônicos para examinar a associação entre o baixo nível de exposição do útero ao arsênico e medidas de crescimento fetal. 
Os resultados encontraram evidências de que a exposição ao baixo nível de arsênico pode estar associada a circunferência reduzida da cabeça de uma forma dependente do sexo que merece uma investigação mais aprofundada.

Este estudo demonstra que dados úteis podem ser extraídos diretamente de registros médicos eletrônicos para a investigação epidemiológica.

\subsubsection{Cross-Hospital Portability of Information Extraction of Cancer Staging Information}

Martinez, D. et al, abordam em seu trabalho a extração de informações de laudos de patologia, com foco em informações codificadas pelo TNM. Os autores defendem que as informações extraídas são essenciais para diagnosticar o grau do câncer em um paciente e para o planejamento de um tratamento individualizado. A extração de tais informações em formato mais estruturado economiza tempo, melhora a comunicação, e sustenta o potencial de suporte automatizado decisão.

\subsubsection{A Text Based Indexing System for Mammographic Image Retrieval and Classification}

Este artigo descreve um sistema de indexação de texto com base em imagens mamográficas para recuperação e classificação de informações. O sistema lida com textos (relatórios estruturados) e imagens (mamografias) para a mineração e classificação. Os laudos médicos são analisados a fim de classificar as imagens mamográficas correspondentes.

\subsubsection{Automatic lymphoma classification with sentence subgraph mining from pathology reports}

Neste trabalho, os autores propõem a utilização de uma ferramenta de mineração de textos para capturar automaticamente as relações de texto narrativo em relatórios médicos de forma não supervisionada, classificando o linfoma de forma similar a um diagnóstico diferencial feito por um patologista.

\subsubsection{The Contribution of the Vaccine Adverse Event Text Mining System to the Classification of Possible Guillain-Barré Syndrome Reports}

Neste artigo, foi desenvolvido um sistema para extrair as principais características dos Relatórios de Eventos Adversos Pós-vacinação. A ferramenta extrai informações fundamentais como diagnóstico, sintomas, tratamento, histórico familiar, histórico médico e causa da morte. Foram analisados em torno de cento e oitenta mil relatórios, onde verificou-se uma classificação eficiente e precisa da Síndrome de Guillian-Barré.

\subsubsection{Construction of an Index of Information From Clinical Practice in Radiology and Imaging Diagnosis}

Serapião $e t$. $a l$. propuseram em seu artigo a criação de um índice de informações sobre a prática da radiologia no idioma português, a fim de melhorar a padronização da linguagem e terminologia médica.

Um total de 61.461 relatórios foram coletados do banco de dados do Sistema de Informação de Radiologia do Hospital das Clínicas - Faculdade de Medicina de Ribeirão Preto, da seguinte forma: 30.000 laudos de raios-x, 27.000 laudos de mamografia e 4.461 laudos de ultrassonografia da tireoide. A técnica de mineração de texto foi aplicada para a seleção de termos e para a construção do índice baseado em uma estrutura de dicionário de sinônimos. O sistema foi criado em * html. 
A mineração de texto resultou em um conjunto de $358.236(\mathrm{n}=100 \%)$ palavras. Desse total, 76.347 termos $(\mathrm{n}=21 \%)$ foram selecionados para formar o índice. Esses termos referem-se à descrição de patologia anatômica, técnicas de imagem, equipamento, tipo de estudo e alguns outros termos compostos. O sistema foi desenvolvido com índice de 78.538 * páginas HTML.

\subsubsection{Natural Language Processing Accurately Categorizes Findings From Colonoscopy and Pathology Reports}

Imler, et. al., afirmam em seu trabalho que pouco se sabe sobre a capacidade de processamento de linguagem natural (PLN) na extração de informações significativas de laudos de gastrenterologia. Neste trabalho os autores utilizaram uma ferramenta open source de PLN para avaliar a precisão dos conceitos clínicos extraídos de 500 laudos de colonoscopia selecionados aleatoriamente. Como resultado da pesquisa realizada, os autores afirmam que o Processamento de Linguagem Natural é capaz de extrair conceitos significativos de laudos médicos com precisão de até $98 \%$.

\section{Considerações Finais}

Este trabalho realizou uma pesquisa utilizando o modelo webibliominig sobre a aplicação da mineração de textos em laudos médicos, afim de obter uma seleção inicial de artigos como núcleo de partida para uma análise de trabalhos mais relevantes referentes ao tema proposto. A pesquisa foi realizada na base Scopus, obtendo resultados satisfatórios, com trabalhos de grande relevância na área pesquisada.

As técnicas da webibliomining demonstraram-se bastante eficientes, proporcionando ao pesquisador uma base fundamental para iniciar uma pesquisa bibliográfica, fundamentando suas pesquisas em trabalhos que possuem credibilidade em meio a comunidade científica e com resultados obtidos a partir de refinamentos, identificando trabalhos com maior números de artigos publicados, autores com maior número de publicações, periódicos com maior número de artigos publicados sobre o tema e a cronologia da produção, identificando os ciclos de maior produção.

A área da saúde tem demonstrado um crescente interesse na mineração de texto. Durante a produção deste trabalho, foram identificados centenas de artigos da área médica voltados para a aplicação de técnicas de mineração de textos, incluindo trabalhos sobre a aplicabilidade da tecnologia de mineração de texto em laudos médicos, tema pesquisado neste artigo.

\section{Referências}

A DAVIS, Matthew et al. Preliminary analysis of in utero low-level arsenic exposure and fetal growth using biometric measurements extracted from fetal ultrasound reports. Environmental Health, v. 14, n. 1, p.49-53, 2015.

BOTSIS, Taxiarchis; WOO, Emilyjane; BALL, Robert F.. The Contribution of the Vaccine Adverse Event Text Mining System to the Classification of Possible Guillain-Barré Syndrome Reports. Applied Clinical Informatics, Rockville, v. 4, n. 1, p.88-99, 2013.

BOZKURT, Selen et al. Using automatically extracted information from mammography reports for decision-support. Journal of Biomedical Informatics, v. 62, p.224-231, ago. 2016.

CAI, Tianrun et al. Natural Language Processing Technologies in Radiology Research and Clinical Applications. Radiographics, v. 36, n. 1, p.176-191, jan. 2016. 
CONFORTO, E. C; AMARAL, D. C.; SILVA, S. L. da. Roteiro para revisão bibliográfica sistemática: aplicação no desenvolvimento de produtos e gerenciamento de projetos. In: Congresso Brasileiro de Gestão de Desenvolvimento de Produto, 8, 2011, 12-14 set, Presidente Prudente-SP. Anais... Presidente Prudente - SP: Faculdade de Tecnologia de Presidente Prudente, 2011. Disponível em:

<http://www.portaldeconhecimentos.org.br/index.php/por/content/view/full/15903>. Acesso em: 12 ago 2016.

COSTA, Helder. Modelo para webibliomining: proposta e caso de aplicação. Rev. FAE, Curitiba, v13, n.1, p. 115-126, jan./jun.2010.

FARRUGGIA, Alfonso; MAGRO, Rosario; VITABILE, Salvatore. A text based indexing system for mammographic image retrieval and classification. Future Generation Computer Systems, Palermo, v. 37, p.243-251, jul. 2014.

HASSANPOUR, Saeed et al. Performance of a Machine Learning Classifier of Knee MRI Reports in Two Large Academic Radiology Practices: A Tool to Estimate Diagnostic Yield. American Journal of Roentgenology, v. 208, n. 4, p.750-753, abr. 2017.

HASSANPOUR, Saeed; LANGLOTZ, Curtis P.. Information extraction from multi-institutional radiology reports. Artificial Intelligence in Medicine, v. 66, p.29-39, jan. 2016.

IMLER, Timothy D. et al. Natural Language Processing Accurately Categorizes Findings From Colonoscopy And Pathology Reports. Clinical Gastroenterology and Hepatology, Indianapolis, v. 11, n. 6, p.689-694, jun. 2013.

KOCBEK, Simon et al. Text mining electronic hospital records to automatically classify admissions against disease: Measuring the impact of linking data sources. Journal of Biomedical Informatics, v. 64, p.158-167, dez. 2016.

LUO, Yuan et al. Automatic lymphoma classification with sentence subgraph mining from pathology reports. Journal of the American Medical Informatics Association, Cambridge, v. 21, n. 5, p.824-832, 2014.

MARTÍNEZ, David S. Hermina et al. Cross-hospital portability of information extraction of cancer staging information. Artificial Intelligence in Medicine, Parkville, v. 62, n. 1, p.11-21, 2014.

RANI, Johanna Johnsi; GLADIS, Dennis; MAMMEN, Joy John. Association analysis of breast cancer data in pathology reports. International Journal of Applied Engineering Research, Chennai, v. 10, n. 69, p.123-128, 2015.

ROY, Partha Pratim et al. Keyword spotting in doctor's handwriting on medical prescriptions. Expert Systems With Applications, v. 76, p.113-128, jun. 2017.

SERAPIÃO, Paulo Roberto Barbosa et al. Construction of an Index of Information From Clinical Practice in Radiology and Imaging Diagnosis. Radiologia Brasileira, Ribeirão Preto, v. 46, n. 5, p.290-298, set. 2013. 